\title{
Distal repair after frozen elephant trunk: open or endovascular?
}

\author{
Christopher K. Mehta ${ }^{1}$, Joseph E. Bavaria ${ }^{2}$ \\ ${ }^{1}$ Division of Cardiac Surgery, Bluhm Cardiovascular Institute, Northwestern University Feinberg School of Medicine, Chicago, IL, USA; ${ }^{2}$ Division \\ of Cardiovascular Surgery, Hospital of the University of Pennsylvania, Philadelphia, PA, USA \\ Correspondence to: Christopher K. Mehta, MD. Division of Cardiac Surgery, Bluhm Cardiovascular Institute, Northwestern University Feinberg \\ School of Medicine, 676 N St Clair St, Arkes Pavilion suite 730, Chicago, IL 60611, USA. Email: Christopher.Mehta@pennmedicine.upenn.edu.
}

Submitted Mar 24, 2020. Accepted for publication Apr 14, 2020.

doi: $10.21037 /$ acs-2020-fet-26

View this article at: http://dx.doi.org/10.21037/acs-2020-fet-26

The frozen elephant trunk (FET) technique has grown in popularity as an effective therapy for aortic arch and descending thoracic aorta pathologies. In particular, many experienced aortic centers have become more aggressive by employing a total arch replacement with FET in the setting of aortic dissection. As a single stage option, the FET stent-graft can be used to cover proximal entry tears in the descending aorta with the goal of inducing false lumen thrombosis and promoting favorable aortic remodeling. If this single stage goal is not achieved, the endograft nonetheless remains a suitable proximal platform onto which a subsequent endovascular or open procedure may be performed when distal reintervention is necessary. Distal aortic reinterventions following FET are common: Kreibich and colleagues demonstrated a 33\% reintervention rate following FET. Common reasons for reintervention included aneurysmal dilation of the descending aorta, endoleak and stent graft-induced new entry tear (SINE) (1). It should be noted that reintervention rate alone is an insufficient measure to judge whether a proximal index operation is successful; ideally a composite of index operation failure, which includes: aortic-related death, unplanned reintervention and aneurysmal diameter $>6 \mathrm{~cm}$, is the metric that should be used to determine the distal success of index procedures.

The development of endovascular stent grafts over the last two decades has led to a paradigm shift in the management of thoracic aortic pathologies. Compared to traditional open thoracoabdominal aortic replacement, endovascular stent-graft deployment leads to quicker patient recovery, lower morbidity and mortality and provides a minimally invasive treatment option for patients who may not be surgical candidates. For these reasons, the endovascular option should be the preferred option for distal reintervention after FET. The FET stent-graft provides an easy platform onto which an endograft can be deployed. Anatomic limitations, including aortic tortuosity, extensive calcification, inadequate access or inadequate landing zones are the biggest hurdles for endovascular therapy. More sophisticated endovascular options are continually becoming available to overcome formerly insurmountable anatomic challenges. In situations where open thoracoabdominal procedures may be needed for distal intervention, the benefit of the FET stent-graft is that it can be clamped and a standard vascular graft can be sewn to the stent-graft portion. This avoids the need for hypothermic circulatory arrest and an open proximal anastomosis in the distal arch. Therefore, the FET stent-graft is a suitable platform for both endovascular and open reinterventions.

The question of whether an endovascular or an open reintervention is better among patients with connective tissue disorders remains controversial. Rylski and colleagues found that patients with Marfan syndrome who underwent Type A aortic dissection repair (at minimal a supracoronary graft) had a $30 \%$ aortic reintervention rate at median followup of 8.4 years (2). Traditionally, open distal procedures have been advocated for patients with connective tissue disorders due to abnormal aortic tissue integrity in these patients. An expert consensus document in 2008 suggested that the radial forces of stent-grafts were not ideal for maintaining aortic tissue integrity and endovascular procedures should be avoided in this patient population (3).

A number of more recent studies have demonstrated promising results for using endovascular therapies in patients with connective tissue disorders. In one of the largest series of stent-graft use in patients with Marfan syndrome, Chen and colleagues challenged the notion that stent-grafts should be avoided in this patient population. In 
this series, 172 patients with Marfan syndrome underwent total arch replacement with FET for both acute and chronic type A dissection, with an operative mortality of $8.1 \%$ and an average follow-up of 6.2 years. False lumen obliteration occurred in $86.1 \%$ of patients at the level of the distal stent-graft, and in 39\% of patients at the unstented middescending thoracic aorta at discharge. Over time, the true lumen expanded at both the distal portion of the stent-graft and the unstented descending aorta, whereas the false lumen decreased in size at the distal stent-graft and remained stable in the unstented distal aorta. At latest follow-up, complete aortic remodeling was achieved in $56.4 \%$ of patients at the level of the distal stent-graft and $28.8 \%$ in the mid-descending thoracic aorta (4). Novel endovascular technologies will inevitably drive more minimally invasive options for second stage reintervention following FET. The next area where we will likely see a burgeoning growth of endovascular use may be in patients with connective tissue disorders, as practitioners become more comfortable using stent-grafts in this patient population. There will always be a role for open procedures in patients with complex anatomic limitations to thoracic endovascular aortic repair (TEVAR); however, as endovascular technologies evolve, these open procedures will likely become less frequently performed.

In summary, the issues regarding open or endovascular repair after FET are as follows:

(I) FET is an excellent TEVAR platform for almost any distal intervention;

(II) FET can serve as a platform for open distal procedures and even simplify these operations in many cases. Therefore, FET should not be considered a contraindication for open reintervention;

(III) The controversy regarding connective tissue disorders presently favors open reconstruction as it is definitive; However, with increasing sophistication of TEVAR, this subset of patients may be treated more aggressively in the future with endovascular options.

\section{Acknowledgments}

None.

\section{Footnote}

Conflicts of Interest: JEB: Terumo Aortic-Primary Investigator, Speakers Bureau. CKM has no conflicts of interest to declare.

Open Access Statement: This is an Open Access article distributed in accordance with the Creative Commons Attribution-NonCommercial-NoDerivs 4.0 International License (CC BY-NC-ND 4.0), which permits the noncommercial replication and distribution of the article with the strict proviso that no changes or edits are made and the original work is properly cited (including links to both the formal publication through the relevant DOI and the license). See: https://creativecommons.org/licenses/by-nc-nd/4.0/.

\section{References}

1. Kreibich M, Berger T, Rylski B, et al. Aortic reinterventions after the frozen elephant trunk procedure. J Thorac Cardiovasc Surg 2019;159:392-9.e1.

2. Rylski B, Bavaria JE, Beyersdorf F, et al. Type A aortic dissection in Marfan syndrome: extent of initial surgery determines long-term outcome. Circulation 2014;129:1381-6.

3. Svensson LG, Kouchoukos NT, Miller DC, et al. Expert consensus document on the treatment of descending thoracic aortic disease using endovascular stent-grafts. Ann Thorac Surg 2008;85:S1-41.

4. Chen Y, Ma WG, Zhi AH, et al. Fate of distal aorta after frozen elephant trunk and total arch replacement for type A aortic dissection in Marfan syndrome. J Thorac Cardiovasc Surg 2018;157:835-49.
Cite this article as: Mehta CK, Bavaria JE. Distal repair after frozen elephant trunk: open or endovascular? Ann Cardiothorac Surg 2020;9(3):226-227. doi: 10.21037/acs-2020-fet-26 